\title{
Investigating the Information Seeking Behaviour of Blind Searchers on the Web
}

\author{
Nuzhah Gooda Sahib \\ Queen Mary University of London \\ London, United Kingdom \\ nuzhah@eecs.qmul.ac.uk
}

\begin{abstract}
I am in the second year of my PhD in the School of Electronic Engineering and Computer Science and my area of research overlaps Information Retrieval and Human-Computer Interaction. In this project, I study the information seeking behaviour of visually impaired searchers and focus on the challenges they face when using online search interfaces. I propose to design and implement an accessible search interface that considers the needs of users of screen readers and also advocate for cognitive load to play an important role in design decisions.
\end{abstract}

Cognitive load, Blind searchers, Information seeking behaviour, Accessibility

\section{INTRODUCTION}

The Web allows access to a large amount of information and this has been a blessing particularly for people with disabilities, for example, visually impaired people. However, the way visually impaired people interact with this wealth of information can pose numerous challenges. Visually impaired people usually access the Web using screen reader software that reads the content of web pages out in computer synthesised speech. The screen reader acts as an intermediary between the user and the graphical user interface, processing web pages sequentially from top to bottom.

This sequential access plays a significant role in how web pages and interfaces are perceived (Andronico et al. 2006a; Stockman and Metatla 2008) and poses a number of challenges for visually impaired users (Andronico et al. 2006a; Borodin et al. 2010) such as lack of context and information overload. In addition, the screen reader interface, like all auditory interfaces, suffers from a lack of persistence. In a search environment, this requires the user to increasingly depend on their memory to keep track of the information they have encountered.

Therefore, in this project, we focus on the information seeking process of blind searchers. We are particularly interested in the challenges they face when using search interfaces which have been designed for sighted searchers. We advocate against transforming interfaces which have been designed for sighted users and propose that completely accessible interfaces should be designed taking into consideration the needs of the visually impaired searchers. We also propose that when designing interfaces, consideration should be given to the cognitive efforts that interface components will require from users of that interface.

\section{RELATED WORK}

The lack of research on the information seeking behaviour of visually impaired searchers have been highlighted in previous work, for example, the NoVA (Non-Visual Access to Digital Libraries) report (Craven and Brophy 2003). In this report, the authors compared how both visually impaired and sighted searchers complete 4 information seeking tasks using online resources. One of the tasks was a search engine task for which the authors reported that visually impaired participants took ten minutes longer than sighted users and that the navigation on the search engine was frustrating (Craven 2004). Despite a lack of previous work on the search behaviour of visually impaired searchers, there have been research efforts (Andronico et al. 2006b; Bigham et al. 2007; Buzzi et al. 2004; Leporini et al. 2004) to address accessibility challenges faced by the visually impaired community. A set of guidelines for designing accessible search interfaces was proposed in (Leporini et al. 2004).

In (Andronico et al. 2006b), Andronico et al. modified the Google interface to improve accessibility while ensuring that the visual appearance of the pages was the same as the original page. They report that the majority of participants thought the interaction with the search 
interface was simplified and a clearer and easier to use interface considerably reduced the time taken to complete their search (Andronico et al. 2006b). The differences in browsing behaviour between visually impaired and sighted users have also been studied in (Bigham et al. 2007) by using a tracking proxy to record participants' interaction in a familiar environment using their own equipment.

\section{RESEARCH PLAN}

Contrary to previous research that are mostly studies of navigation and browsing behaviour, in this project, we focus on the behaviour of visually impaired searchers for the complete information seeking process, that is, from the query formulation stage to results management. In addition, we study complexsearch tasks as they are challenging, cognitively intensive and affect searchers' performance. We are particularly interested in the challenges posed by the mode of interaction and how a lack of visual cues impacts the information seeking behaviour of visually impaired searchers. Hence, the overall aim of this project is to address these challenges and our plan is to design and implement a search interface that is accessible as well as usable for visually impaired searchers.

Therefore, we have identified the following objectives for this project:

- Address the gap in literature by conducting studies on the information seeking behaviour of visually impaired searchers. Such investigations will provide valuable insight to enhance the design of accessible search interfaces. A comparative analysis with the information seeking behaviour of sighted searchers will also allow us to define how a lack of visual cues impacts the search behaviour of users of screen readers.

- Develop a framework to guide the development of accessible information seeking interfaces. Following findings from our comparative analysis of the information seeking behaviour between sighted and visually impaired searchers, we can make recommendations to guide the design of accessible search interfaces.

- Design search interface components that are accessible as well as usable for visually impaired users. As visually impaired searchers already work in cognitive intensive conditions due to their use of screen readers (Chandrashekar et al. 2006), we aim to design search interface support features that do not require a high level of cognitive effort.

- Evaluate the implemented interface. We aim to carry out both a performance-based evaluation and cognitive-based evaluation to determine whether our accessible search interface meets the needs of our target users.

\section{CURRENT STAGE}

We have recently completed two user studies where we compare the information seeking behaviour of 15 blind and 15 sighted searchers while they complete complex search tasks online. As we wanted to observe participants in settings as close to those in which they performed their day to day search activities, participants used their own equipment and chose their own search tasks. To ensure that tasks were of an appropriate level of complexity, we provided participants with guidelines to choose their own complex tasks. Participants used their own equipment and their interaction with the search engine was recorded. We collected additional information through a demographic questionnaire and a semi-structured interview.

We carried out a comparative analysis between the groups of searchers and identified significant differences at various stages of the information seeking process, for example, query formulation, results exploration etc.

\section{EXPECTED GAINS FROM ATTENDING THE DOCTORAL CONSORTIUM}

During the doctoral consortium, I would like to discuss the following with the panel and other research students:

- Discuss issues regarding the development of a framework to measure the cognitive load imposed by user interfaces. There has been several attempts in the past to measure cognitive load, for example, NASA TLX (Hart and Staveland 1988). These methods do not perform very well for measuring the cognitive load of interfaces. However, to design interface components that are accessible and usable, we need to be able to measure the cognitive effort required by different interface designs. To do so, we require a framework that can be used with different groups of users (both sighted and visually impaired) to effectively measure the cognitive load associated with the use of an interface. Implementing a framework suited for visual and auditory interfaces is a challenging problem which would benefit many other studies in studies of human-computer interaction. Thus, I would like to discuss this with participants at the doctoral consortium and I believe it 
would be incredibly helpful to get others' expert opinions on this problem.

- Following the initial findings from the observational studies I recently completed (described in section 4), I would like to consult members of the panel on their thoughts for an accessible search interface for complex information seeking.

- I would also like to discuss methodological issues regarding the analysis of data collected during the observational studies as well aschallenges in how to perform statistical testing on such data.

While I am looking forward to discussing the abovementioned issues with the panel, I am also interested to hear and contribute ideas towards the projects of other research students.

\section{REFERENCES}

Patrizia Andronico, Marina Buzzi, Carlos Castillo, and Barbara Leporini. Improving search engine interfaces for blind users: a case study. Universal Access in the Information Society, 5(1):23-40, 2006a.

Patrizia Andronico, Marina Buzzi, Barbara Leporini, and Carlos Castillo. Testing google interfaces modified for the blind. In Proceedings of the $15^{\text {th }}$ international conference on World Wide Web, pages 873-874. ACM, 2006b.

Jeffrey P. Bigham, Anna C. Cavender, Jeremy T. Brudvik, Jacob O.Wobbrock, and Richard E. Lander. WebinSitu: a comparative analysis of blind and sighted browsing behaviour. In Proceedings of the 9th international ACM SIGACCESS conference on Computers and accessibility, pages 51-58. ACM, 2007.
Yevgen Borodin, Jeffrey P. Bigham, Glenn Dausch, and I. V. Ramakrishnan. More than meets the eye: a survey of screen-reader browsing strategies. In Proceedings of the 2010 International Cross Disciplinary Conference onWeb Accessibility (W4A), pages 1-10. ACM, 2010.

Marina Buzzi, Patrizia Andronico, and Barbara Leporini. Accessibility and usability of search engine interfaces: preliminary testing. In Proceedings of ERCIM UI4ALL Workshop, 2004.

Sambhavi Chandrashekar, Tony Stockman, Deborah Fels, and Rachel Benedyk. Using think aloud protocol with blind users: a case for inclusive usability evaluation methods. In Proceedings of the 8th international ACM SIGACCESS conference on Computers and accessibility, pages 251-252. ACM, 2006.

Jenny Craven. Linear searching in a non-linear environment: The information seeking behaviour of visually impaired people on the world wide web. In Computers Helping People with Special Needs, page 626. 2004.

Jenny Craven and Peter Brophy. Non-visual access to the digital library: the use of digital library interfaces by blind and visually impaired people. Technical report 145, CERLIM, Manchester, 2003.

S. G Hart and L. E Staveland. Development of NASA-TLX (Task load index): Results of empirical and theoretical research. Human mental workload, 1:139-183, 1988.

Barbara Leporini, Patrizia Andronico, and Marina Buzzi. Designing search engine user interfaces for the visually impaired. In Proceedings of the 2004 international cross-disciplinary workshop on Web accessibility (W4A), pages 57-66, New York City, New York, 2004. ACM

Tony Stockman and Oussama Metatla. The influence of Screen-Readers on web cognition. In Proceedings of the Accessible Design in the Digital World Conference, 2008. 